\title{
Therapeutic mechanisms and routes of delivery of mesenchymal stem cells in veterinary medicine: A point of view
}

\author{
AMANDA BARACHO TRINDADE HILL ${ }^{1,2, *}$; JoNATHAN EDwiN BARACHO TRINDADE HILL ${ }^{2}$ \\ ${ }^{1}$ Centro Universitário Max Planck, Indaiatuba, Brazil \\ 2 CellTech-Stem Cell Technologies, Campinas, Brazil
}

Key words: Cell therapy, Application, Injection, Pharmacodynamics, Pharmacokinetics

\begin{abstract}
Mesenchymal stem cells (MSCs) represent an important tool in veterinary regenerative medicine due to their ability to home to injury sites and secrete molecules that regulate niches into regenerative microenvironments. Successful cell therapy depends on many factors, including choice of administration route and application of understanding of cell potency and their therapeutic mechanisms. In this point of view, the authors leverage the tumultuous history of the field to demonstrate the need for clinicians to continually update themselves as new discoveries are made in order to avoid misalignments in the future, especially regarding administration routes and dose frequency, as well as to explore recent insights into MSC plasticity, therapeutic mechanisms, and cell delivery systems.
\end{abstract}

\section{Introduction}

Approximately 30 years ago, mesenchymal stem cells (MSCs) were first defined as cells that could be isolated from adult tissue, grow in vitro, and differentiate into mesodermal lineages (Caplan, 1991). Since then, mesenchymal stem cells have been widely studied due to their therapeutic potential, and it is difficult to identify an aspect of our understanding of stem cells that has not changed dramatically since their discovery. From where -and indeed if- they may be found in adult organisms, to how they cause their therapeutic effects, to what their full range of differentiation potential is, to how they function in healthy versus diseased microenvironments, and of course how best to apply them in a clinical context, the history of our knowledge of the field is nothing if not fraught with reversals of opinion upon revelation of further evidence. As the level of interest and quantity of studies performed in the area continue to increase, it is critical to synthesize new and more efficient clinical techniques from the results of this research. Our objective here is to discuss and give our point of view regarding some of the changes that have impacted the understanding of MSCs' therapeutic mechanisms and the effect that delivery routes have on them.

*Address correspondence to: Amanda Baracho Trindade Hill, Amanda.baracho@prof.unieduk.com.br

Received: 07 August 2021; Accepted: 06 October 2021

\section{Point of View}

The first report of stem cell isolation after birth was published in 1966. The cells in this report, derived from bone marrow, were plastic-adherent, had fibroblast-like morphology, and could form colonies when seeded in tissue culture (Friedenstein et al., 1966). These cells were able to undergo osteogenic, chondrogenic and adipogenic differentiation (Friedenstein et al., 1966). Not until 30 years later were fibroblast-like cells isolated from umbilical cord tissue (McElreavey et al., 1991). The beliefs that MSCs could only be found in select adult tissues and only differentiate into mesodermal lineages persisted for approximately another decade until the isolation of a novel adult stem cell population, from adipose tissue, was reported (Zuk et al., 2002). It is now widely accepted that adult stem cells exist in all tissues due to their physiological function, which is to contribute to tissue growth, regeneration, and healing throughout life (Dong et al., 2015). The cells isolated from adipose tissue were able to differentiate not only into osteogenic, chondrogenic and adipogenic lineages, which are mesodermal germ layer lineages, but also into neurogenic cells, which are part of the ectodermal germ layer (Zuk et al., 2002). Two years later, another group achieved differentiation of MSCs into hepatic cells, which are part of the endodermal lineage (Lee et al., 2004) These breakthroughs proved that MSCs could differentiate into lineages from all three germ layers and could only correctly be defined as pluripotent cells and not multipotent. Cells that are considered multipotent 
are those with restricted ability to differentiate (Biehl and Russell, 2009). This was a paradigm shift in the stem cell field, as only embryonic stem cells were previously thought to have such wide differentiation potential.

The previously held assumption (still held by some of the field's old guard) that MSCs were unable to differentiate into ectodermal and endodermal lineages was made due to a lack of understanding of the mechanics of differentiation, not a lack of differentiation ability on the part of the cells. What paved the way for the demonstration of their pluripotency was the discovery of the factors necessary to induce MSCs to differentiate into cells from all three germ lineages. The gradual revelations of the range of tissues in which MSCs may be found and their true range of differentiation capacity are just two examples of the prevalence of incorrect conclusions that were drawn in the area due to a lack of investigation rather than convincing evidence. It is our duty as scientists to be open to new discoveries that overturn old ideas, and through this openness not allow our experiments and therapies to stagnate.

Another watershed event in the field was the progress in understanding how chronic disease states affect stem cell niches. The fate of endogenous MSCs is typically determined by microenvironmental factors in specific niches, comprising cellular and noncellular components from both local and systemic sources, that regulate stem cell survival, location, proliferation, potency and differentiation (Jones and Wagers, 2008). This regulation is achieved through several signaling pathways, such as physical and structural stimuli, and neural, humoral, paracrine, autocrine, and metabolic interactions (Scadden, 2007). In developmental, healing, or diseased states, different microenvironmental signals are generated, regulating stem cell fate (Martinez-Agosto et al., 2007). For example, when tissues are damaged, MSCs are naturally released into circulation, where they migrate to the injury site and secrete molecules that foster a microenvironment that promotes regeneration (Chapel et al., 2003; Caplan, 2009), functioning as growth factors and regenerative molecule storage.

Although many factors that dictate cell fate, including neighboring cells, the extracellular matrix, soluble molecules, and physical stimuli, have been discovered and investigated (Dong et al., 2015), it is still not clear how most somatic stem cell niches work (Fuchs et al., 2004). What is clear, however, is that stem cell niches gradually lose their functionality in a state of continuous disease (Dong et al., 2015). It has been shown, for example, that patients with chronic diseases such as diabetes, chronic renal failure, and arterial or venous insufficiency have impaired cell migration, reduced growth factor production, and poor tissue remodeling on an organism-wide level, displaying chronic wounds (Dong et al., 2015). The inference can be made that these chronic wounds are at least partially caused and/or exacerbated by the ineffective migration and growth factor production of endogenous MSCs, which are integral to the healing process.

Therefore, the administration of stem cells to the patient to "help" the tissue that is currently impaired to heal can be understood as a restoration of the functionality of affected niches. This is why patients suffering from chronic diseases are those most helped by stem cell therapy: the administered cells are able to restore the functionality of affected niches. Indeed, it is well known that, in adequate microenvironments, stem cells can improve cell survival and amplify paracrine effects, which favour trophic support, improve homing to the lesion site, and promote suppression of inflammatory factors and immune responses to promote functional recovery (Discussed in Dong et al., 2015).

Regarding the method by which MSCs are able to alter the functionality of microenvironments, it is now well accepted that the therapeutic effects of MSC administration are related to their paracrine activity (Gnecchi et al., 2008; Zhang et al., 2012; Park et al., 2018). These paracrine effects are achieved when the bioactive factors secreted by the administered MSCs stimulate the patient's own resident stem cells to build the relevant new tissue (Caplan, 2017). This stands in contrast to the previous model, as described by Caplan (2017), in which it was assumed that the medical benefits of MSCs were derived from their differentiation into tissue-producing, regenerating cells. To illustrate the significance of this evolution in understanding, the very same author that proposed the name "mesenchymal stem cells" in 1991 now urges that the name be changed to "medicinal signaling cells" in order to reflect the cells' two main functions: the abilities to home to injury sites and to secrete bioactive immunomodulatory and trophic (regenerative) factors, effectively meaning that MSCs make and administer therapeutic drugs in situ that are medicinal in nature (Caplan, 2017). We could therefore be justified in defining these cells as biological drugs due to their ability to provide the necessary supplementation of biological factors in contribution to the resident cells' tissue regeneration efforts. Indeed, MSCs have already been called "multidrug dispensaries or drugstores" (Caplan and Correa, 2011).

This modern understanding of how MSCs achieve their therapeutic effects and how microenvironments react to chronic disease states allows us to make the following general inference: if a patient has a chronic condition (Dong et al., 2015), is elderly, or has a large injury, their natural supply of MSCs, though present and somewhat active, is being inefficiently employed by the body and must therefore be supplemented (Caplan and Correa, 2011) in order to correct the disease state.

Now that the nature of MSCs, their role in the adult organism, and the general framework under which they achieve their therapeutic effects are better understood in light of recent research, we can apply this improved understanding to clinical applications, specifically to administration routes. At the present time, intravenous (IV) cell administration is the most studied route, as well as the route most used in clinical trials for systemic diseases, such as kidney failure, in which, until 2021, only reports of IV administration of MSCs had been published (Quimby et al., 2011; Quimby et al., 2013; Yun and Lee, 2019). Recently, application by subcutaneous injection has been reported, and results have been promising (Figueiredo et al., 2021). Though effective in many cases, IV administration entails several well-documented inefficiencies that could be eliminated by updating administration routes using current knowledge of cell behavior.

Foremost among these inefficiencies is the fact that, as many studies have demonstrated, MSCs become trapped in 
the lungs immediately after intravenous injection (IV). Some of those cells undergo apoptosis, some return to general circulation, and only a small fraction of MSCs are found to survive, migrate to and engraft into the target organs, as reviewed in Wang et al. (2016). The cells that are able to move to other major organs, such as the liver or the kidneys, are no longer detectable in the body after a variable but short period (24 h to 14 days), as reviewed in Masterson et al. (2021). Additionally, there is evidence that MSCs in general are shortlived after IV infusion (Eggenhofer et al., 2012). It has also been proposed that this initial pulmonary trapping of stem cells might alter their tissue homing ability as well (Fischer et al., 2009). As it is now known that MSCs exert their therapeutic effects through paracrine activity, the inference can be made that shorter survival in situ and reduced homing effectiveness are deleterious to the strength of the effect that the administered cells exert on the relevant tissue microenvironments and, therefore, that administration routes that maximize cell survivability and homing effectiveness should be preferred, supporting the rationale that cell pharmacodynamics are integrally linked to the pharmacokinetics of administration routes.

In order to investigate the clinical validity of this inference, we may consider Braid et al. (2018), in which MSC persistence was compared between IV, intramuscular (IM), intraperitoneal (IP) and subcutaneous (SC) delivery routes. The authors found that MSCs were able to survive for 5 months post IM injection in the muscle, but only 1-4 weeks post injection when delivered IV, IP, or SC. Additionally, to verify the clinical relevance of this information, we summarize a report by Giri and Galipeau (2020) that compared three delivery routes: IV, IP and SC, to treat colitis, and found that animals that received cells via the IP or SC routes showed more beneficial effects than those that received via IV. They concluded that, at least for colitis, IP and SC are better delivery methods than IV, begging the question of whether this may be true in general (Giri and Galipeau, 2020).

Moreover, Creane et al. (2017) demonstrated that DNA from MSCs was still present at injection sites 3 months after IM injection, though the MSCs themselves could not be detected in any internal organs. This same paper also demonstrated that the cells were able to reduce inflammation in areas distant from the injection site, even though they did not distribute themselves throughout the body (Creane et al., 2017). This can be explained by the fact that muscle is a highly vascularized tissue, allowing the bioactive and trophic factors released by the cells to be distributed systemically even when the cells themselves in large part remain close to the injection site, supporting the model in which MSCs affect injury sites through their paracrine activity (Hamidian Jahromi and Davies, 2019).

These studies illustrate that IM administration delivers greater cell persistence and, as a result, greater therapeutic effects when compared to IV administration. The IM route would be an especially attractive method of cell delivery for systemic diseases, as we can infer from Creane et al. (2017) showing that the cells can continue secreting bioactive factors for a longer period of time and that those factors would affect injury sites distant from the injection site. In addition to this primary benefit, IM administration carries the auxiliary benefit of being less invasive and, in the context of veterinary medicine, less costly and less risky to the patient. This would, in turn, result in a reduction of costs, which could be passed through to owners as a more accessible dollar value per administration.

Lastly, another clinically relevant finding described in Giri and Galipeau (2020) was that of the comparison of single versus repeated doses. The authors determined that repeated doses were more beneficial (Giri and Galipeau, 2020). In this context, it has been proposed that repeated cell administrations have cumulative beneficial effects and, as a result, are notably more effective than a single administration due to their persistent paracrine effects. Wysoczynki et al. (2018) corroborates, finding that the effect of a high single dose of cells is less beneficial than the cumulative effects of three smaller doses.

\section{Conclusion}

As we can see from the findings presented, the history of our understanding of mesenchymal stem cells has changed drastically in numerous ways over the past half century and continues to be revolutionized as new discoveries are made. The authors of this paper urge researchers and clinicians in the area to adapt a forward-thinking mindset and be open to applying and investigating the results of these discoveries in their own work, as well as making a commitment to staying apace of developments in the field. As numerous authors cited in this communication have demonstrated, such a change can only lead to better outcomes for our patients. Recent research clearly supports the use of IM administration over IV from both theoretical and practical perspectives, and this change would not only increase the effectiveness of each administration but also result in secondary benefits in terms of invasiveness and cost in the context of veterinary medicine.

Authors' Contribution: ABTH contributed to the conception and design of the manuscript, analyzed and interpreted literature and drafted the manuscript. JEBTH contributed to the design of the manuscript and revised it for important intellectual content. All authors read and approved the final manuscript.

Funding Statement: This manuscript received no specific grant from any funding agency in the public, commercial, or not-for-profit sectors.

Conflicts of Interest: The authors declare that they have no conflicts of interest to report regarding the present manuscript.

\section{References}

Biehl JK, Russell B (2009). Introduction to stem cell therapy. The Journal of cardiovascular nursing 24: 98.

Braid LR, Wood CA, Wiese DM, Ford BN (2018). Intramuscular administration potentiates extended dwell time of mesenchymal stromal cells compared to other routes. Cytotherapy 20: 232-244. DOI 10.1016/j.jcyt.2017.09.013.

Caplan AI (1991). Mesenchymal stem cells. Journal of Orthopaedic Research 9: 641-650. DOI 10.1002/(ISSN)1554-527X.

Caplan AI (2009). Why are MSCs therapeutic? New data: New insight. Journal of Pathology 217 318-324. 
Caplan AI (2017). Mesenchymal stem cells: Time to change the name!. Stem Cells Translational Medicine 6: 1445-1451. DOI 10.1002/sctm.17-0051.

Caplan AI, Correa D (2011). The MSC: An injury drugstore. Cell Stem Cell 9: 11-15. DOI 10.1016/j.stem.2011.06.008.

Chapel A, Bertho JM, Bensidhoum M, Fouillard L, Young RG, Frick J, Demarquay C, Cuvelier F, Mathieu E, Trompier F et al. (2003). Mesenchymal stem cells home to injured tissues when co-infused with hematopoietic cells to treat a radiation-induced multi-organ failure syndrome. Journal of Gene Medicine 5: 1028-1038.

Creane M, Howard L, O'Brien T, Coleman CM (2017). Biodistribution and retention of locally administered human mesenchymal stromal cells: Quantitative polymerase chain reaction-based detection of human DNA in murine organs. Cytotherapy 2017: 384-394. DOI 10.1016/j.jcyt.2016.12.003.

Dong L, Hao H, Han W, Fu X (2015). The role of the microenvironment on the fate of adult stem cells. Science China Life Sciences 58: 639-648. DOI 10.1007/s11427-0154865-9.

Eggenhofer E, Benseler V, Kroemer A, Popp F, Geissler E, Schlitt H, Hoogduijn MJ (2012). Mesenchymal stem cells are shortlived and do not migrate beyond the lungs after intravenous infusion. Frontiers in Immunology 3: 297. DOI 10.3389/fimmu.2012.00297.

Figueiredo DG, Figueiredo JPG, Valeriano ACD, Rodrigues DF, Hill JEBT, Hill ABT (2021). Mesenchymal stem cell injection into kidney acupoints: A case report of A feline with chronic kidney disease. Biomedical Journal of Scientific \& Technical Research 38: 1-3.

Fischer UM, Harting MT, Jimenez F, Monzon-Posadas WO, Xue H, Savitz SI, Cox CS (2009). Pulmonary passage is a major obstacle for intravenous stem cell delivery: The pulmonary first-pass effect. Stem cells and Development 18: 683-692. DOI 10.1089/scd.2008.0253.

Friedenstein AJ, Piatetzky-Shapiro II, Petrakova KV (1966). Osteogenesis in transplants of bone marrow cells. Development 16: 381-390.

Fuchs E, Tumbar T, Guasch G (2004). Socializing with the neighbors: Stem cells and their niche. Cell 116: 769-778. DOI 10.1016/ S0092-8674(04)00255-7.

Giri J, Galipeau J (2020). Mesenchymal stromal cell therapeutic potency is dependent upon viability, route of delivery, and immune match. Blood Advances 4: 1987-1997. DOI 10.1182/bloodadvances.2020001711.

Gnecchi M, Zhang Z, Ni A, Dzau VJ (2008). Paracrine mechanisms in adult stem cell signaling and therapy. Circulation Research 103: 1204-1219. DOI 10.1161/CIRCRESAHA.108.176826.

Hamidian Jahromi S, Davies JE (2019). Concise review: Skeletal muscle as a delivery route for mesenchymal stromal cells. Stem Cells Translational Medicine 8: 456-465. DOI 10.1002/sctm.18-0208.

Jones DL, Wagers AJ (2008). No place like home: Anatomy and function of the stem cell niche. Nature Reviews Molecular Cell Biology 9: 11-21.
Lee KD, Kuo TKC, Whang-Peng J, Chung YF, Lin CT, Chou SH, Lee OKS (2004). In vitro hepatic differentiation of human mesenchymal stem cells. Hepatology 40: 1275-1284. DOI 10.1002/(ISSN)1527-3350.

Masterson CH, Tabuchi A, Hogan G, Fitzpatrick G, Kerrigan SW, Jerkic M, Curley GF (2021). Intra-vital imaging of mesenchymal stromal cell kinetics in the pulmonary vasculature during infection. Scientific Reports 11: 1-12.

Martinez-Agosto JA, Mikkola HKA, Hartenstein V, Banerjee U (2007). The hematopoietic stem cell and its niche: A comparative view. Genes \& Development 21: 3044-3060.

McElreavey KD, Rvine AI, Ennis KT, Mclean WI (1991). Isolation, culture and characterisation of fibroblast-like cells derived from the Wharton's jelly portion of human umbilical cord. Biochemical Society Transactions 19: 29S. DOI 10.1042/ bst019029s.

Park WS, Ahn SY, Sung SI, Ahn JY, Chang YS (2018). Strategies to enhance paracrine potency of transplanted mesenchymal stem cells in intractable neonatal disorders. Pediatric Research 83: 214-222. DOI 10.1038/pr.2017.249.

Scadden DT (2006). The stem-cell niche as an entity of action. Nature 441: 1075-1079.

Quimby JM, Webb TL, Habenicht LM, Dow SW (2013). Safety and efficacy of intravenous infusion of allogeneic cryopreserved mesenchymal stem cells for treatment of chronic kidney disease in cats: Results of three sequential pilot studies. Stem Cell Research \& Therapy 4: 1-12. DOI 10.1186/scrt198.

Quimby JM, Webb TL, Gibbons DS, Dow SW (2011). Evaluation of intrarenal mesenchymal stem cell injection for treatment of chronic kidney disease in cats: A pilot study. Journal of Feline Medicine and Surgery 13: 418-426. DOI 10.1016/j. jfms.2011.01.005.

Wang H, Liang X, Xu ZP, Crawford DH, Liu X, Roberts MS (2016). A physiologically based kinetic model for elucidating the in vivo distribution of administered mesenchymal stem cells. Scientific Reports 6: 1-12. DOI 10.1038/srep22293.

Wysoczynki M, Khan A, Bolli R (2018). New paradigms in cell therapy: Repeated dosing, intravenous delivery, immunomodulatory actions, and new cell types. Circulation Research 123: 138158. DOI 10.1161/CIRCRESAHA.118.313251.

Yun CW, Lee SH (2019). Potential and therapeutic efficacy of cellbased therapy using mesenchymal stem cells for acute/ chronic kidney disease. International Journal of Molecular Sciences 20: 1619-1615. DOI 10.3390/ijms20071619.

Zhang S, Chen L, Liu T, Zhang B, Xiang D, Wang Z, Wang Y (2012). Human umbilical cord matrix stem cells efficiently rescue acute liver failure through paracrine effects rather than hepatic differentiation. Tissue Engineering Part A 18: 13521364. DOI 10.1089/ten.tea.2011.0516.

Zuk PA, Zhu M, Ashjian P, de Ugarte DA, Huang JI, Mizuno H, Hedrick MH (2002). Human adipose tissue is a source of multipotent stem cells. Molecular Biology of the Cell 13: 4279-4295. DOI 10.1091/mbc.e02-02-0105. 